\title{
ZIGOMICOSIS CUTANEA: REPORTE DE CASO
}

\section{CUTANEOUS ZYGOMYCOSIS: CASE REPORT}

\author{
Farfán Gutiérrez Christian ${ }^{1,2, a}$ Arce Lazo Marco²b
}

\section{RESUMEN}

La zigomicosis cutánea es una infección micótica causada por zigomicetos que afecta la piel. Se produce mayormente en los pacientes diabéticos no controlados y los individuos inmunodeprimidos. Tiene 2 formas clínicas: zigomicosis cutánea primaria y zigomicosis cutánea secundaria. La primera se caracteriza por lesiones necróticas e inoculación del hongo por lo general por un traumatismo. Si se diagnostica a tiempo, tiene un buen pronóstico. Zigomicosis secundaria suele ser una complicación y extensión de la variedad rinocerebral que comienza como una fístula palpebral y progresa a una lesión necrótica con un mal pronóstico. El diagnóstico se realiza mediante la identificación del hongo mediante examen directo, cultivo y biopsia. El tratamiento para la enfermedad primaria es el desbridamiento quirúrgico más anfotericina B. El tipo secundario se trata con anfotericina B y/o posaconazol. Presentamos un caso de un adulto mayor sin antecedentes contributorios que presentó zigomicosis cutánea y fue tratado con éxito en nuestro servicio.

\section{Palabras clave}

Zigomicosis, micosis, antimicóticos. (Fuente: DeCS)

\section{ABSTRACT}

Zygomycoses skin is a fungal infection that affects the skin zygomycetes. It occurs in uncontrolled diabetics patients and immunocompromised individuals. It has two clinical forms: cutaneous primary and secondary skin. The first is characterized by necrotic lesions and inoculation of the fungus usually by trauma. If diagnosed early, usually it has a good prognosis. Zygomycoses secondary is usually a complication and extent of rhinocerebral variety starts as a lid fistula and progresses to a necrotic lesion with a poor prognosis. The diagnosis is made by identifying the fungus by direct examination, culture and biopsy. The treatment for primary disease is the most amphotericin B. The secondary type surgical debridement is treated with amphotericin B and / or posaconazole. We present a case of an adult without contributory history that I present skin zygomycoses and was treated in our service.

\section{Keywords}

Zygomycosis, mycoses, antifungal agents . (Source: MeSH NLM)

${ }^{1}$ Hospital Regional Honorio Delgado - Arequipa.

2 Universidad Mayor de San Marcos.

a Residente de cuarto año de Cirugía Bucal y Maxilofacial.

${ }^{\mathrm{b}}$ Cirujano Bucal y Maxilofacial.

\section{Correspondencia:}

Farfán Gutiérrez Christian

Correo electrónico: christianjr_88_@hotmail.com
Este es un artículo Open Access distribuido bajo la licencia Creative Commons Atribución-NoComercialCompartirlgual 4.0 


\section{INTRODUCCIÓN}

Zigomicosis, también conocido como mucormicosis (debido a su taxonomía), es causada por un grupo de hongos oportunistas de la clase de zigomicetos ${ }^{(1}$ $2,3)$. Se presenta principalmente en pacientes diabéticos no controlados $y$ en individuos inmunodeprimidos. Sus variedades clínicas son rinocerebral (la más frecuente), pulmonar, cutánea, gastrointestinal y diseminada.

Zigomicosis cutánea tiene dos formas: cutánea primaria y cutánea secundaria. Cutánea primaria, que comienza con varios tipos de trauma, es frecuente en pacientes inmunodeprimidos y tiene un buen pronóstico si se diagnostica a tiempo. Zigomicosis cutánea secundaria, que es por lo general una difusión a la piel desde una ubicación rinocerebral, se produce con mayor frecuencia en los pacientes diabéticos no controlados e individuos inmunocomprometidos, siendo una entidad invasora grave con un mal pronóstico(4) ${ }^{(4)}$ No es posible estimar las tasas exactas de incidencia de zigomicosis, porque la mayoría de los datos provienen de informes de casos o series de $\operatorname{casos}^{(5,6)}$. Hay, sin embargo, algunos datos, principalmente de pacientes hematológicos, los cuales indican que la incidencia de zigomicosis ha aumentado en los últimos años ${ }^{(7)}$.

El diagnóstico de zigomicosis cutánea es a menudo difícil debido a los resultados no específicos de la infección. El clínico debe tener un alto grado de minuciosidad y utilizar todas las herramientas de diagnóstico disponibles, ya que el diagnóstico temprano conduce a un mejor resultado. Indicios de una posible zigomicosis cutánea incluyen lesiones necróticas en la piel de un paciente inmunodeprimido o un paciente inmunocompetente víctima de un trauma o una quemadura.

Si una herida no se cura adecuadamente, a pesar del uso correcto de los antibióticos, o si aparece una inflamación en sus bordes, se debe sospechar zigomicosis entre otros diagnósticos. La biopsia de las lesiones y cultivo es necesaria para establecer el diagnóstico. El tratamiento de zigomicosis consiste en el desbridamiento quirúrgico, uso de fármacos antifúngicos y la reversión de los factores de riesgo subyacentes, si es posible. El desbridamiento quirúrgico debe repetirse cuantas veces sea necesario. El principal fármaco antifúngico utilizado en el tratamiento de zigomicosis es anfotericina $\mathrm{B}^{\left({ }^{(8)}\right.}$. El pronóstico de zigomicosis cutánea es mejor que el de las otras formas de zigomicosis, pero no es despreciable.

\section{REPORTE DE CASO}

Paciente varón de 64 años que aproximadamente hace 08 meses nota un aumento de volumen en la cara, que al pasar el tiempo va disminuyendo y que queda como una lesión negra. Acude a Centro de Salud donde le prescriben Clindamicina, $300 \mathrm{mg}$ VO por 07 días, y le informan que tiene una infección al hueso por lo que lo refieren a nuestro hospital para su tratamiento.

En el examen clínico extraoral existe una asimetría facial por una aumento de volumen con predominio de región geniana superior izquierda y una lesión pigmentada plana, de bordes irregulares, consistencia firme, base amplia, de color negro marrón, de más o menos $2,5 \mathrm{~cm} \times 1,5 \mathrm{~cm}$, ubicada en la región geniana superior izquierda, indolora a la palpación.

En la evaluación intraoral se evidencia un leve aumento de volumen a nivel de tabla vestibular de pieza dentaria 2.2 que presenta una lesión cariosa compatible con una necrosis pulpar y necrosis pulpar de pieza 2.7. Al realizar la evaluación tomográfica, evidenciamos una imagen hipodensa con borde definidos a nivel apical de pieza 2.2 y

2.7. Tanto la evaluación clínica como imagenológica nos llevan al posible diagnóstico de osteomielitis maxilar, por lo cual se programa la exodoncia de la pieza 2.2 y 2.7, curetaje alveolar, desbridamiento y exéresis de lesión a nivel facial. 

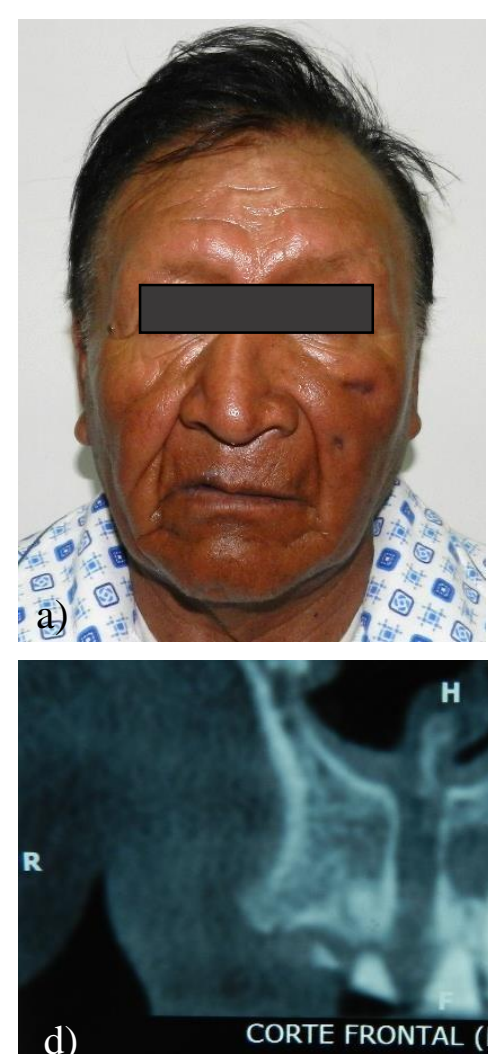
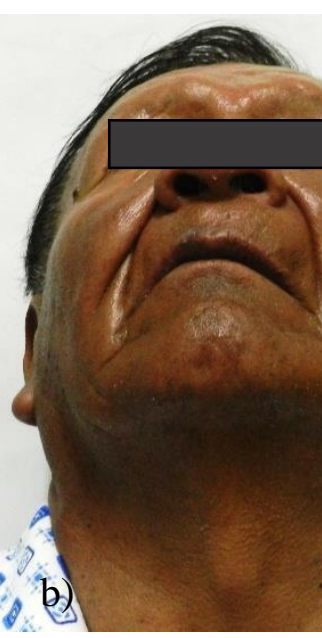


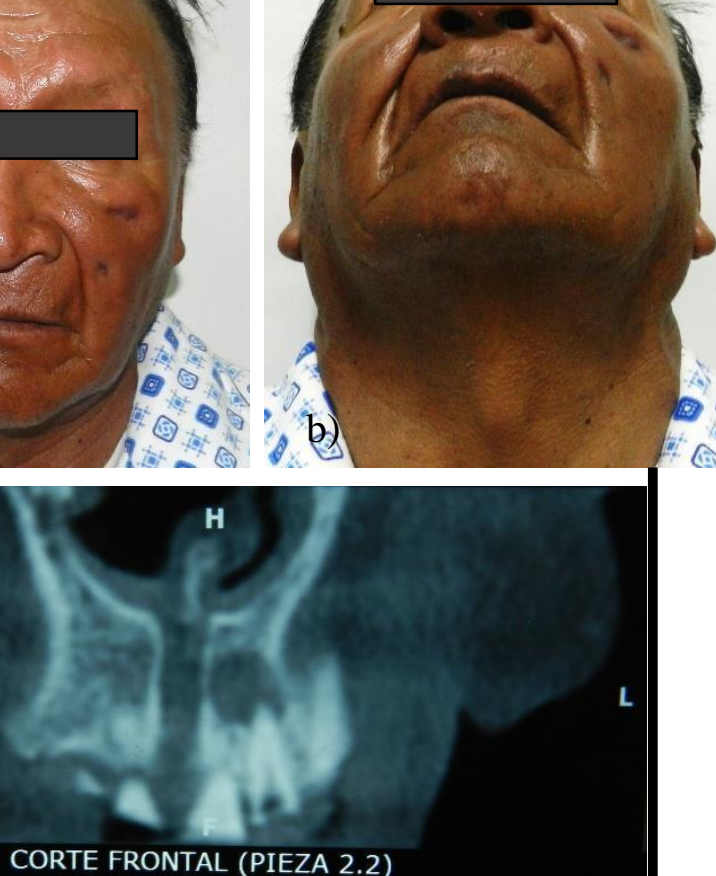

CORTE FRONTAL (PIEZA 2.2)
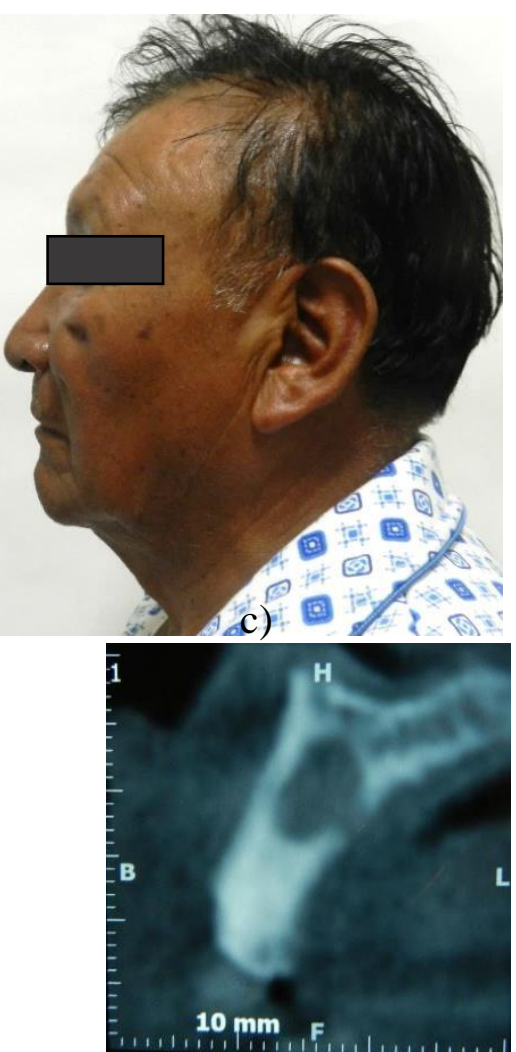

H

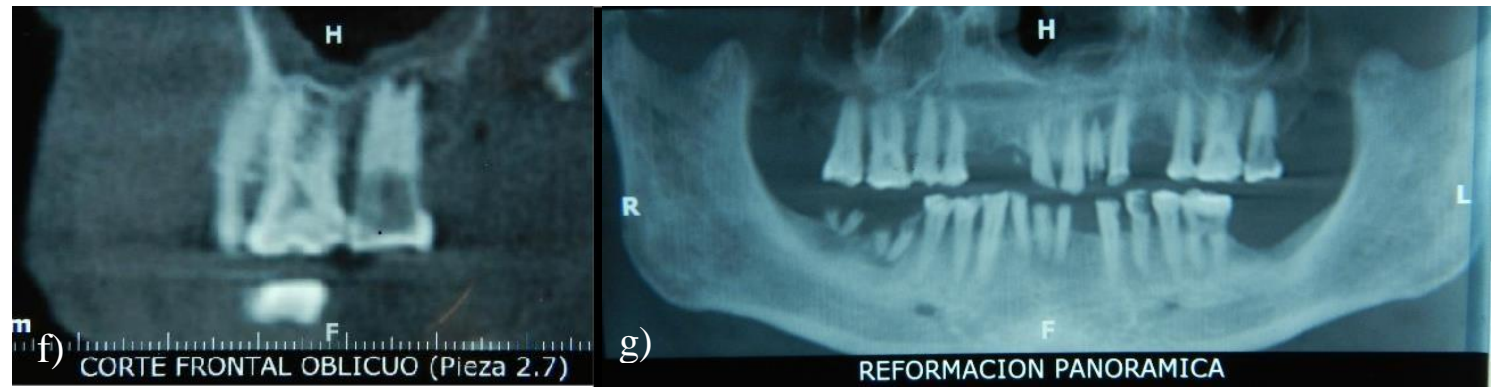

Figura 1: a) b) y c) Examen extraoral que evidencia aumento de volumen y lesión pigmentaria a nivel de region geniana superior izquierda; d) y e) corte coronal y sagital a nivel de pieza dentaria 2.2 que muestra imagen hiperdensa compatible con lesión periapical; f) corte coronal a nivel de pieza 2.7 con lesión periapical; g) corte panorámico que muestra lesión pericapical a nivel de pieza dentaria 2.2 y 2.7 como probables vías de acceso de infección micótica.

Se realiza la exodoncia de la pieza 2.2 y 2.7 , curetaje alveolar y la exeresis y debridamiento de la lesion pigmentada en cara enviándose a anatomía patológica. En la evaluacion microscópica evidencia necrosis, abscedación y formación de granulomas atribuible a infección tipo zigomicosis con presencia de hifas y esporas. Se aumenta al debridamiento quirúrgico ya realizado el antimicótico Itroconazol, $100 \mathrm{mg}$ cada 24 horas por 06 meses.

El paciente evoluciona favorablemente y se realizan controles posteriores donde no se evidencia recidiva de lesión. 

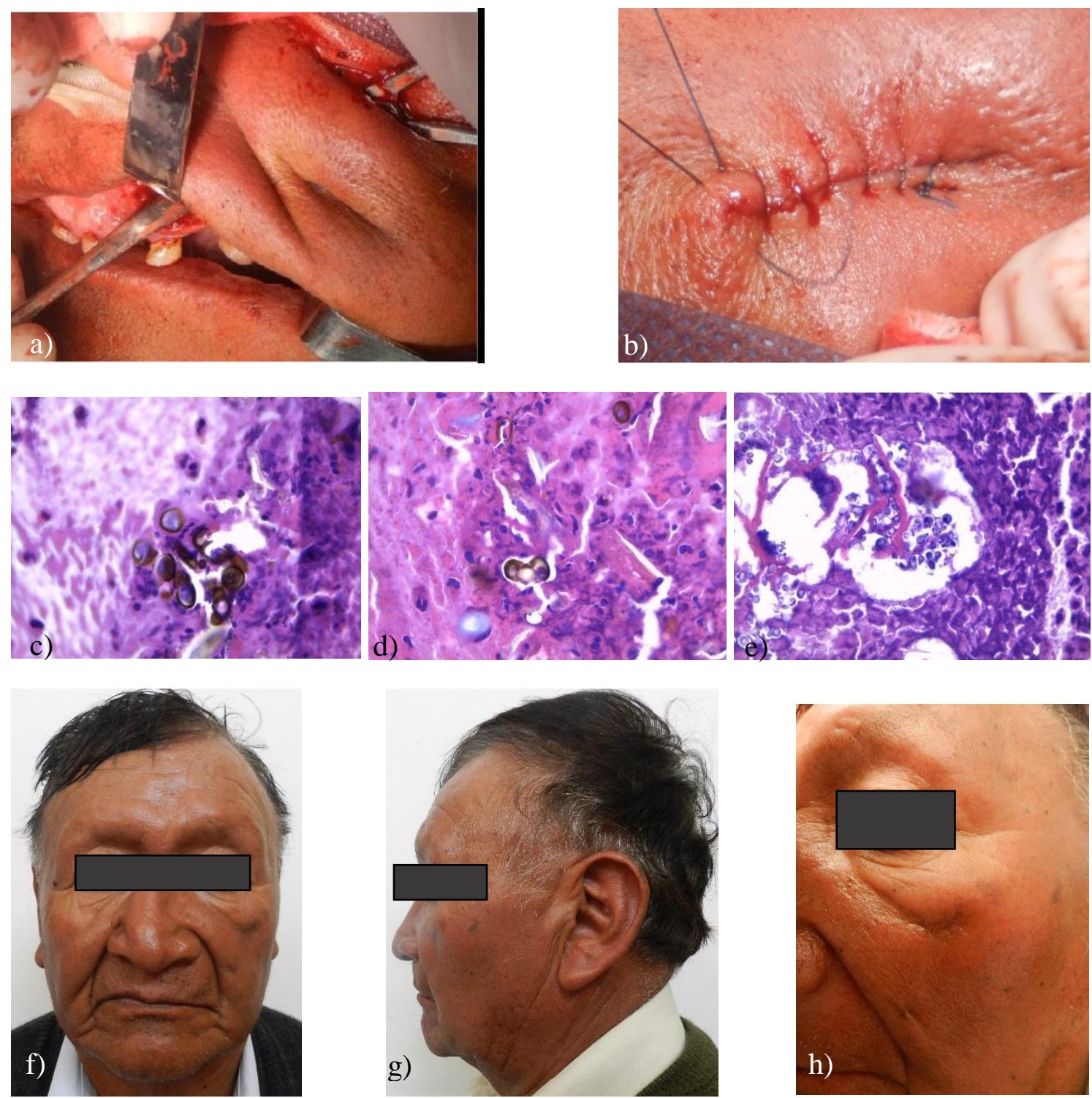

Figura 2: a) y b) desbridamiento y exéresis de lesión a nivel de región geniana superior izquierda; c),

d) y e) cortes histopatológicos que evidencian la proliferación de esporas e hifas compatibles con zigomicosis; f), g) y h) evaluación extraoral posterior a 4 meses del acto quirúrgico donde se evidencia herida operatoria en proceso óptimo de cicatrización.

\section{DISCUSIÓN}

Zigomicosis fue descrita por primera vez como una causa de enfermedad en humanos en 1885 , y el primer caso a nivel cutáneo se informó en $1929^{(9)}$.

La Zigomicosis cutánea es la tercera forma más común de zigomicosis (11-19\%) después de la rinocerebral (39\%) y la pulmonar $(24 \%){ }^{(10-12)}$. A diferencia de otras presentaciones, el $40-50 \%$ de los pacientes con zigomicosis cutánea son inmunocompetentes $(10,11)$. El trauma es el factor predisponente más común para zigomicosis cutánea, especialmente cuando se asocia con la suciedad. Los factores de riesgo para zigomicosis cutánea incluyen la diabetes no controlada, cetoacidosis diabética, quemaduras, insuficiencia renal, transplante de órganos sólidos crónicos, hemopatías malignas, neutropenia, el uso de 
esteroides, el uso prolongado de voriconazol y deferoxamina y el bajo peso al nacer ${ }^{(12,13)}$.

Zigomicosis cutánea puede presentarse como una enfermedad localizada, profunda o diseminada $^{(14)}$. Casi la mitad de los pacientes con zigomicosis cutánea tiene su enfermedad confinada en los tejidos cutáneos y subcutáneos, mientras que la enfermedad puede extenderse a los tejidos más profundos, tales como los huesos, los tendones y los músculos en el $24 \%$ de los casos. La inoculación directa de la piel después de un traumatismo penetrante, cirugía, quemaduras, accidentes de tráfico, caídas y picaduras de insectos es el puerto de entrada habitual para esta enfermedad(12). El contacto con el suelo o la vegetación que contiene zygomycetes aumenta la posibilidad de contraer la enfermedad $^{(15)}$. En nuestro paciente no fue posible determinar si el trauma principal en la cara o una infección secundaria crónica en una pieza dentaria causó la enfermedad del paciente. El tratamiento de zigomicosis cutánea incluye una combinación de desbridamiento quirúrgico, terapia antifúngica efectiva y resolución de los factores de riesgo subyacentes. Una tasa de mortalidad de menos de $10 \%$ ha sido reportada para los pacientes con mucormicosis cutánea después del tratamiento con desbridamiento agresivo y terapia antifúngica adjunta $^{(11)}$.

En conclusión, podemos manifestar que debemos recordar a la zigomicosis como una alternativa diagnóstica en pacientes con lesiones pigmentarias a nivel cutáneo con antecedentes de trauma o, en nuestro caso, con infección crónica odontogénica para realizar el correcto manejo para el beneficio de nuestros pacientes.

Contribuciones de autoría: FGC y ALM participaron en la revisión de la literatura, diagnóstico y tratamiento del paciente. Ambos autores redactaron y aprobaron el artículo.

Fuente de financiamiento: autofinanciado.

Conflictos de interés: Los autores declaran no tener conflictos de interés en la publicación de este artículo.

\section{REFERENCIAS BIBLIOGRÁFICAS}

1. Bonifaz A. Zigomicosis. Micología médica básica, $3^{\underline{a}}$ ed. México: McGraw-Hill; 2009.

2 Eucker J, Sezer O, Graf B, Possinger K. Mucormycosis. Mycoses. 2001; 44:253-60.
3. Brown J. Zygomycosis: an emerging fungal infection. Am J Health Syst Pharm. 2005; 62: 2593-6.

4. Chander J, Kaur J, Attri A, et al. Primary cutaneous zygomycosis from a tertiary care centre in north-west India. Indian J Med Res. 2010; 131: 765-70.

5. Saegeman V, Maertens J, Meersseman W, Spriet I, Verbeken E, Lagrou K. Increasing incidence of mucormycosis in university hospital, Belgium. Emerg Infect Dis. 2010; 16:1456-8.

6. Skiada A, Pagano L, Groll A, et al, for the European Confederation of Medical Mycology Working Group on Zygomycosis. Zygomycosis in Europe. Analysis of 230 cases accrued by the registry of the European Confederation of Medical Mycology (ECMM) Working Group on Zygomycosis between 2005 and 2007. Clin Microbiol Infect. 2011;17: 1859-67.

7. Bitar D, Van Cauteren D, Lanternier $F$, et al. Increasing incidence of Zygomycosis (mucormycosis), France, 1997-2006. Emerg Infect Dis. 2009; 15:1395401.

8. Spellberg B, Edwards Jr J, Ibrahim A. Novel perspectives on mucormycosis: pathophysiology, presentation and management. Clin Microb Rev. 2005; 18:556-69.

9. Paltauf A. Mycosis mucorina: ein Beitrag zur Kenntnis der menschilchen Fadenpiltzer-krankungen. Virchows Arch Pathol Anat 1885; 102:543-64.

10. Skiada A, Petrikkos G. Cutaneous zygomycosis. Clin Micro- biol Infect. 2009; 15(5):41-5.

11. Roden MM, Zaoutis TE, Buchanan WL, Knudsen TA, Sark- isova TE, Schaufele RL, et al. Epidemiology and outcome of zygomycosis: a review of 929 reported cases. Clin. Infect Dis. 2005; 41:634-53.

12 Spellberg B, Edwards Jr J, Ibrahim A. Novel perspectives on mucormycosis: pathophysiology, presentation, and management. Clin. Microbiol. Rev. 2005; 18:556-69.

13. Immunodeficient infant: a case report and review of literature. Ann Plast Surg. 2008; 60:433-6.

14. Skiada A, Petrikkos G. Cutaneous zygomycosis. Clin Micro- biol Infect. 2009; 15(5):41-5.

15. Chakrabarti A. Cutaneous zygomycosis: major concerns. Indian J. Med. Res. 2010; 131: 739-41. 